\title{
Cryoballoon Pulmonary Vein Isolation with Real-Time Recordings from the Pulmonary Veins
}

\author{
K. R. JULIAN CHUN, M.D., ${ }^{*}$ ALEXANDER FÜRNKRANZ, M.D., ${ }^{*}$ ANDREAS METZNER, M.D., \\ BORIS SCHMIDT, M.D., ROLAND TILZ, M.D., THOMAS ZERM, M.D., ILKA KÖSTER, M.D., \\ DIETER NUYENS, M.D., ERIK WISSNER, M.D., FEIFAN OUYANG, M.D., \\ and KARL HEINZ KUCK, M.D.
}

From the Department of Cardiology, Asklepios Klinik St. Georg, Hamburg, Germany

\begin{abstract}
Pulmonary Vein Conduction During Cryoballoon Ablation. Introduction: Cryoballoon (CB) ablation represents a novel technology for pulmonary vein isolation (PVI). We investigated feasibility and safety of CB-PVI, utilizing a novel spiral catheter (SC), thereby obtaining real-time PV potential registration.

Methods: Following double transseptal puncture, a Lasso catheter (Biosense Webster, Diamond Bar, CA, USA) and the $28 \mathrm{~mm} \mathrm{CB}$ were positioned within the left atrium. A novel SC (Promap, ProRhythm Inc., Ronkonkoma, NY, USA) was inserted through the lumen of the CB allowing PV signal registration during treatment. Time to PV conduction block was analyzed. If no stable balloon position was obtained, the SC was exchanged for a regular guide wire and PV conduction was assessed after treatment by Lasso catheter.

Results: In 18 patients, 39 of 72 PVs (54\%) were successfully isolated using the SC. The remaining 33 PVs were isolated switching to the regular guide wire. Time to PV conduction block was significantly shorter in PVs in which sustained PVI was achieved as compared to PVs in which PV conduction recovered within 30 minutes ( $33 \pm 21$ seconds vs $99 \pm 65$ seconds). In $40 \mathrm{PV}$ s, time to PV conduction block was not obtained because of: (1) PVI not being achieved during initial treatment; (2) a distal position of the SC; or (3) isolation with regular guide wire. No procedural complications occurred.

Conclusion: Visualization of real-time PV conduction during CB PVI is safe, feasible, and allows accurate timing of PVI onset in a subset of PVs. Time to PV conduction block predicts sustained PVI. However, mechanical properties of the SC need to be improved to further simplify CB PVI. (J Cardiovasc Electrophysiol, Vol. 20, pp. 1203-1210, November 2009)
\end{abstract}

atrial fibrillation, pulmonary vein isolation, catheter ablation, cryoablation

\section{Introduction}

Catheter ablation using radiofrequency current (RFC) for pulmonary vein isolation (PVI) is a well-established therapy option for the treatment of paroxysmal atrial fibrillation (PAF). ${ }^{1}$ Applying contiguous left atrial (LA) linear lesions using "RFC point-by-point" burning is technically complex and typically requires a three-dimensional (3D) reconstruction system. ${ }^{2}$ In addition, the application of RFC energy is related to complications such as PV stenosis, stroke, atrioesophageal fistula, and "man-made" atrial tachyarrhythmias. ${ }^{3-6}$ In contrast, the novel cryoballoon system (Cryocath, Montreal, Canada) simplifies $\mathrm{PVI}^{7-10}$ and atrial appendage isolation ${ }^{11}$ solely based on fluoroscopy. However, a second transseptal puncture for the placement

\footnotetext{
*Both authors contributed equally to this manuscript.
}

Dr. Kuck reports serving as consultant for European Advisory Board Cryocath, Montreal, Canada. Dr. Chun reports honoraria relevant to this topic.

Address for correspondence: K. R. Julian Chun, M.D., Department of Cardiology, Asklepios Klinik St. Georg, Lohmühlenstr. 5, 20099 Hamburg, Germany. Fax: 49-40-1818-85-4435; E-mail: jongichun@t-online.de

Manuscript received 25 March 2009; Revised manuscript received 21 April 2009; Accepted for publication 6 May 2009.

doi: $10.1111 / \mathrm{j} .1540-8167.2009 .01549 . \mathrm{x}$ of a diagnostic spiral catheter (Lasso, Biosense Webster, Diamond Bar, CA, USA) is still required to evaluate the endpoint of PVI after each cryoenergy application. Utilizing a single transseptal LA access the cryoballoon would need to be removed for the Lasso catheter after each freeze, increasing the risk of air embolism and/or damaging the balloon. Currently, the operator has no means to assess the effect of cryoballoon ablation on PV conduction during the freezing period. Thus, real-time PV potential registration during the freeze, knowledge of precise mode of PV potential sequence change, and assessment of the exact time of PVI may improve the understanding of cryoballoon lesion formation and enhance procedural efficacy. Therefore, we investigated feasibility and safety of cryoballoon PVI, utilizing instead of the guide wire a novel spiral catheter (SC, Promap, ProRhyrthm Inc., Ronkonkoma, NY, USA) originally designed for high-intensity focused ultrasound (HIFU) balloon ablation ${ }^{12}$ that allows for the first-time real-time PV potential recording during the freeze.

\section{Methods}

\section{Inclusion and Exclusion Criteria}

A total of 18 patients with PAF were included in this study and provided written informed consent. Inclusion criteria were, a history of highly symptomatic PAF $(\geq 1$ episode/week) despite treatment with $\geq 1$ antiarrhythmic drug (AAD) and availability of the novel investigational 
spiral catheter (SC) (Promap, ProRhythm). Exclusion criteria were defined as a LA diameter $\geq 55 \mathrm{~mm}$, severe left ventricular hypertrophy ( $\mathrm{LV}$ wall thickness $\geq 15 \mathrm{~mm}$ ), reduced left ventricular ejection fraction ( $\mathrm{EF}<55 \%)$, LA thrombus, prior stroke, or heart failure. No preprocedural imaging for patient selection was performed.

\section{Cryoballoon Catheter and Ablation Procedure}

The concept of the "single big cryoballoon" technique for PVI (Arctic Front, Cryocath ${ }^{\mathrm{TM}}, 28 \mathrm{~mm}$ diameter, 10.5F shaft) has been described in detail elsewhere. ${ }^{9}$ A central lumen within the catheter is used for (1) the insertion of the SC or guide wire and (2) injection of contrast medium during PV angiography. All procedures were performed under conscious sedation using boluses of midazolam, fentanyl, and a continuous infusion of propofol $(1 \%)$. Following double transseptal puncture using the modified Brockenbrough technique, two 8F sheaths (SL1; St. Jude Medical, Minneapolis, MN, USA) were positioned within the LA. Thereafter, heparin was repeatedly administered to maintain an activated clotting time of 250 to 300 seconds. Selective PV angiography was performed to identify the PV anatomy using standard projections ( $\mathrm{RAO} 30^{\circ}, \mathrm{LAO} 40^{\circ}$ ). Left common PVs were defined according to Marom et al. ${ }^{13}$ and treated utilizing the "cross-talk technique" as previously described. ${ }^{9}$ Baseline PV potentials were recorded using a conventional computerized EP system (AXIOM Sensis, Siemens, Forchheim, Germany). The anterior sheath was exchanged for a $12 \mathrm{~F}$ transseptal sheath (FlexCath, Cryocath) over which the cryoballoon $(28 \mathrm{~mm})$ catheter was introduced into the LA. Both transseptal sheaths were continuously flushed with heparinized saline. To assess the exact position of the inflated $28-\mathrm{mm}$ balloon in relation to the PV ostium, contrast medium (Imeron, diluted with saline $0.9 \%$ at 1:1 ratio) was injected from the distal lumen of the cryoballoon catheter. The phrenic nerve $(\mathrm{PN})$ was continuously paced $(10 \mathrm{~V}, 2.9 \mathrm{~ms})$ from the superior caval vein during energy delivery along the right PVs. Throughout each energy application, the position of the cryoballoon was verified. No other intraprocedural imaging except fluoroscopy was used. After the 30-minute waiting period, 1 extra freeze was applied to all PVs.

\section{Spiral Catheter: Two in One-PV Potential Recording and Guide Wire}

Instead of the regular guide wire, a novel thin SC (15 $\mathrm{mm}$, shaft diameter: $0.9 \mathrm{~mm}$, length: $143 \mathrm{~cm}, 6$ electrodes, ProRhythm) was introduced into the central lumen of the 28 $\mathrm{mm}$ cryoballoon catheter. The SC has a very soft distal shaft and ends with a circular spiral loop carrying 6 electrodes allowing PV potential recordings. The SC served (1) as a guide wire to navigate the cryoballoon to the PVs and to give support for a stable balloon position at the PV ostium. In addition, the SC was used (2) for real-time recording of LA-PV conduction during the freezing period. The time interval between start of the cryoballoon freeze and block of PV conduction (s) as well as the corresponding cryoballoon temperature $\left({ }^{\circ} \mathrm{C}\right)$ was measured. To exclude additive cryothermal effects, determination of time to PV conduction block was only eligible for PVs in which PVI was achieved with the first freeze. If the $\mathrm{SC}$ had to be placed in a far distal position within the PV to stabilize the balloon, no PV potential was recorded and time to PV conduction block could not be de- termined. In the latter case, LA-PV conduction was assessed after the freeze using the Lasso catheter. If no stable balloon position at the PV ostium could be achieved, the SC was exchanged for a regular guide wire (stiff wire, Amplatz, Cook, Bloomington, IN, USA) and PV conduction was assessed with the Lasso catheter following each cryoballoon application as previously described. ${ }^{9}$ Sustained PVI was defined as PVI extending the 30 minute waiting period after PVI. Nonsustained PVI was defined as recovery of PV conduction within the 30 minute waiting period as documented by either Lasso or SC.

\section{Post Ablation Treatment}

All patients underwent postprocedural transthoracic echocardiography and chest X-ray to rule out a pericardial effusion or pneumothorax. After the procedure, all patients were treated with intravenous heparin (target PTT 50-70 seconds). Oral anticoagulation (phenprocoumon targeting an INR value of 2.0-3.0 for at least 3 months) was initiated the following day.

\section{Endpoints}

\section{Primary endpoint}

Feasibility was defined as successful sustained PVI utilizing the novel SC catheter in conjunction with the cryoballoon.

\section{Secondary endpoints}

Secondary endpoints were defined as: (1) ability to assess PV signal quality during the freeze; (2) time interval from the start of the freeze to the moment of PV conduction block (s) provided PVI was achieved with the first cryoballoon application; and (3) procedure-related complications.

\section{Statistical Analysis}

Data mean \pm standard deviation (SD) was used to describe continuous variables with normal distribution. The Student $t$-test was performed to calculate differences between groups. Groups of ordinal data were compared by the Friedman test, a nonparametric rank sum test for repeated measurements. Because of the small data set, the $P$ value computation was based on the exact method. A P value $<0.05$ was considered statistically significant.

\section{Results}

\section{Patients}

Among 18 patients (14 males, mean age: $62 \pm 8$ years) enrolled in this initial feasibility and safety study (Table 1), all had a history of PAF with a mean duration of $6 \pm 5$ years and were refractory to AAD treatment $(2 \pm 1)$. All patients had a normal left ventricular ejection fraction, 9 patients had a history of hypertension and 2 patients had a known coronary artery disease (Table 1).

\section{Selective Pulmonary Vein Angiographies}

Selective PV angiography was used to identify PVs and to calculate mean PV diameters. In 18 patients, a total of $72 \mathrm{PVs}$ including $2 \mathrm{LSPV}$ and $2 \mathrm{LIPV}$ branches of 2 left common PVs 
TABLE 1

Patient Demographics

\begin{tabular}{|c|c|c|c|c|c|c|c|c|}
\hline Patient & Gender & $\begin{array}{c}\text { Age } \\
\text { (Years) }\end{array}$ & $\begin{array}{c}\text { Concomittant } \\
\text { Heart } \\
\text { Disease }\end{array}$ & Hypertension & LVEF & $\begin{array}{c}\text { LA } \\
\text { Size } \\
(\mathbf{m m})\end{array}$ & $\begin{array}{c}\text { Duration } \\
\text { of PAF } \\
\text { (Years) }\end{array}$ & $\begin{array}{c}\text { Number (n) } \\
\text { of Failed } \\
\text { AAD }\end{array}$ \\
\hline 1 & Male & 69 & No & Yes & Normal & 44 & 2 & 2 \\
\hline 2 & Male & 67 & No & Yes & Normal & 54 & 4 & 2 \\
\hline 3 & Male & 67 & No & Yes & Normal & 41 & 6 & 2 \\
\hline 4 & Female & 57 & No & No & Normal & 42 & 2 & 1 \\
\hline 5 & Male & 58 & No & Yes & Normal & 40 & 3 & 1 \\
\hline 6 & Male & 55 & No & No & Normal & 45 & 3 & 2 \\
\hline 7 & Male & 66 & No & Yes & Normal & 43 & 1 & 2 \\
\hline 8 & Male & 44 & No & No & Normal & 41 & 4 & 2 \\
\hline 9 & Male & 65 & No & No & Normal & 35 & 12 & 1 \\
\hline 10 & Male & 63 & CAD & No & Normal & 41 & 9 & 2 \\
\hline 11 & Female & 73 & CAD & Yes & Normal & 35 & 10 & 2 \\
\hline 12 & Male & 51 & No & No & Normal & 46 & 2 & 1 \\
\hline 13 & Female & 71 & No & No & Normal & 42 & 4 & 2 \\
\hline 14 & Male & 59 & No & No & Normal & 37 & 2 & 2 \\
\hline 15 & Female & 60 & No & Yes & Normal & 36 & 5 & 1 \\
\hline 16 & Male & 63 & No & Yes & Normal & 41 & 10 & 1 \\
\hline 17 & Male & 68 & No & No & Normal & 38 & 20 & 2 \\
\hline 18 & Male & 68 & No & Yes & Normal & 44 & 6 & 1 \\
\hline \multicolumn{2}{|c|}{ MEAN } & 62 & - & - & - & 41 & 6 & 2 \\
\hline \multicolumn{2}{|c|}{ STD } & 8 & - & - & - & 5 & 5 & 1 \\
\hline \multicolumn{2}{|c|}{ Total (n) } & - & 2 & 9 & - & - & - & - \\
\hline
\end{tabular}

$\mathrm{AAD}=$ antiarrhythmic drug; $\mathrm{LV} \mathrm{EF}=$ left ventricular ejection fraction; $\mathrm{CAD}=$ coronary artery disease; PAF $=$ paroxysmal atrial fibrillation; $\mathrm{LA}=$ left atrium; STD = standard deviation.

$(28.2 \pm 0.5 \mathrm{~mm})$ with a short common trunk were identified. Mean PV diameter was $19.8 \pm 3.2 \mathrm{~mm}$ for LSPVs $(\mathrm{n}=18)$, $17.3 \pm 2.5 \mathrm{~mm}$ for LIPVs $(\mathrm{n}=18), 19.2 \pm 2.4 \mathrm{~mm}$ for RSPVs $(\mathrm{n}=18)$ and $19.4 \pm 3.5 \mathrm{~mm}$ for RIPVs $(\mathrm{n}=18)$.

\section{Procedural Data}

Mean procedure and fluoroscopy times including PN pace mapping were $209 \pm 45$ minutes and $34.1 \pm 13.8$ minutes, respectively. The total balloon time (defined as time from insertion to removal of the cryoballoon from the LA) was $120 \pm 25$ minutes. A mean of $147 \pm 37 \mathrm{~mL}$ contrast medium was required for PV angiographies. Successful acute PVI was achieved in all PVs (72/72 PVs, 100\%).

\section{Primary Endpoint}

Spiral catheter as a guide wire for cryoballoon pulmonary vein isolation

A total of 39 of 72 PVs (54\%) were successfully isolated using the cryoballoon in conjunction with the SC (Fig. 1). In 4 of 39 PVs, SC-guided PVI was feasible, however, lacking real-time PV potential visualization due to a very distal position of the SC electrodes (Table 2, Fig. 1). If the SC was utilized for cryoballoon ablation, successful PVI correlated with PV location: LSPVs (17/18, 94\%); LIPVs (11/18, 61\%); $\operatorname{RSPV}(8 / 18,44 \%)$; RIPV (3/18, 17\%); $\mathrm{P}<0.001$. Thus, success of LSPV isolation was significantly higher, utilizing the SC when compared to other PVs. All remaining 33 of 72 PVs (46\%) were isolated after switching to the regular stiff guide wire (Table 3, Fig. 1). The reason to switch to the conventional stiff guide wire was the inability to achieve a stable cryoballoon position.

\section{Secondary Endpoints}

PV signal quality during the cryoballoon freeze

Real-time visualization of PV conduction during a cryoballoon freeze could be obtained in 38 of 72 (53\%) PVs. In all 38 PVs, good quality SC PV signals were noted during the cryoballoon freeze allowing for PV potential visualization and LA-PV conduction assessment (Figs. 1 and 3A,B). 32 of $72(44 \%)$ PVs were eligible for determination of time to PV conduction block (Figs. 1 and 3A,B). In the remaining $40 \mathrm{PVs}$, time to PV conduction block could not be determined because: (1) no PV isolation was achieved during the first cryoballoon application (4 PVs); (2) no PV potential was recorded due to a distal SC position (4 PVs); (3) no PVI was achieved with the SC (2 PVs); or (4) only the regular guide wire was used for ablation because of unstable balloon positions using the SC (30 PVs) (Fig. 1).

\section{Time to PV conduction block}

A total of $32 \mathrm{PVs}$ were eligible for time to PV conduction block analysis (Fig. 1). Time to PV conduction block was significantly shorter in PVs with sustained PVI (18 PVs) as compared to PVs with recovered PV conduction (14 PVs): $33 \pm 21$ seconds vs $99 \pm 65$ seconds, $\mathrm{P}=0.002$. Temperatures did not differ at time of PVI $\left(-32 \pm 12^{\circ} \mathrm{C}\right.$ vs $-35 \pm 13^{\circ} \mathrm{C}, \mathrm{P}=$ n.s.) (Table 4 ). Recovery of PV conduction occurred for all 14 PVs within the first 3 minutes after termination of cryothermal energy delivery.

\section{Procedural complications}

No procedural complications were observed. 


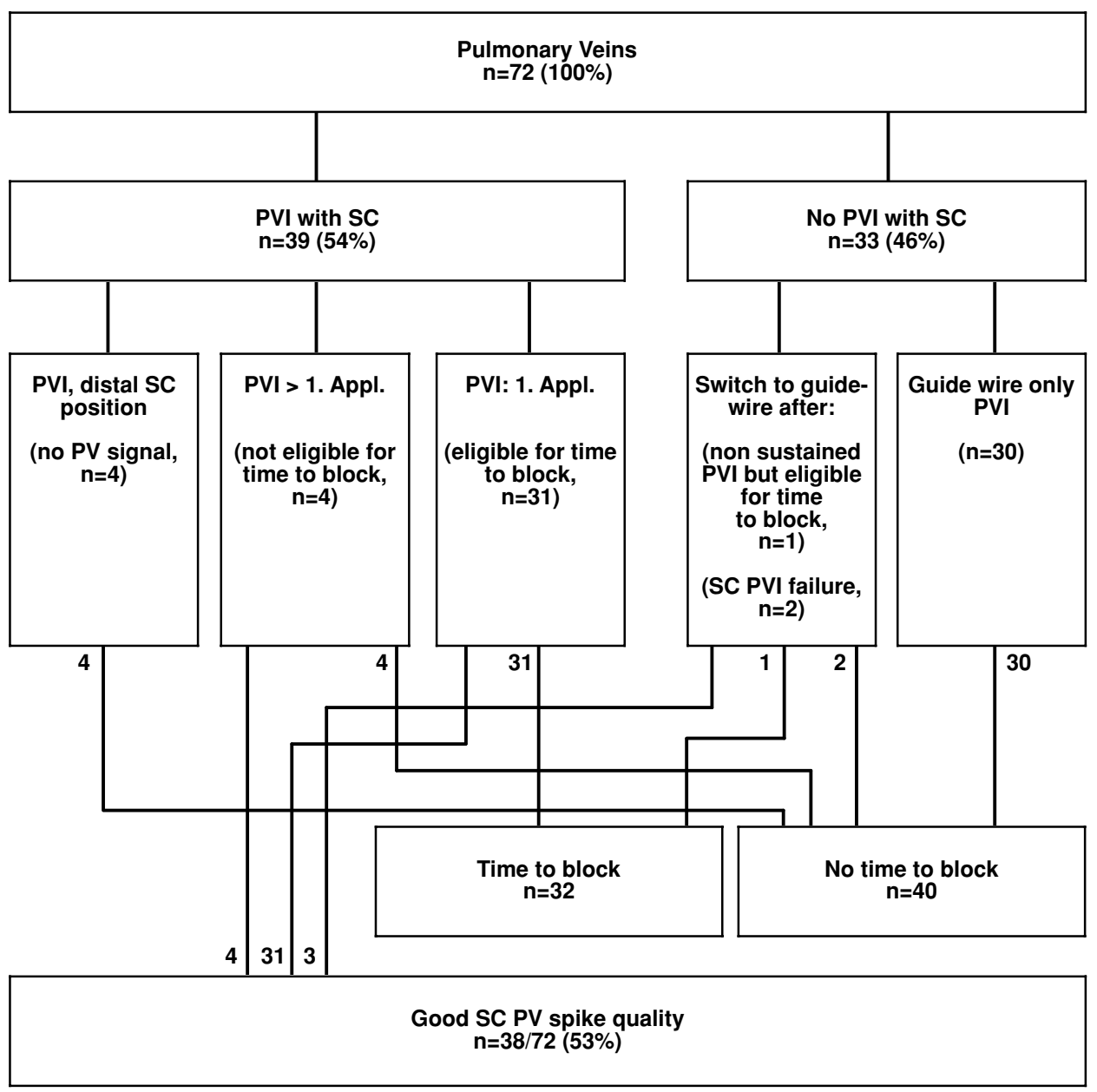

Figure 1. Flowchart: overview of all pulmonary veins. PVI-pulmonary vein isolation, PV-pulmonary vein, SC-spiral catheter.

TABLE 2

Cryoballoon PVI with Novel Spiral Catheter: Real-Time PV Potential Visualization

\begin{tabular}{|c|c|c|c|c|c|c|c|c|}
\hline \multirow[b]{2}{*}{ Patient } & \multicolumn{2}{|r|}{ LSPV } & \multicolumn{2}{|r|}{ LIPV } & \multicolumn{2}{|r|}{ RSPV } & \multicolumn{2}{|r|}{ RIPV } \\
\hline & PVI (s) & Temperature $\left({ }^{\circ} \mathbf{C}\right)$ & PVI (s) & Temperature $\left({ }^{\circ} \mathrm{C}\right)$ & PVI (s) & Temperature $\left({ }^{\circ} \mathrm{C}\right)$ & PVI (s) & Temperature $\left({ }^{\circ} \mathrm{C}\right)$ \\
\hline 1 & $20^{*}$ & -54 & Wire & Wire & $95^{*}$ & $-54^{*}$ & $16^{*}$ & -20 \\
\hline 2 & $29^{*}$ & -40 & $33^{*}$ & -40 & $11^{*}$ & $-14^{*}$ & Wire & Wire \\
\hline 3 & $18^{*}$ & -43 & Wire & Wire & Wire & Wire & Wire & Wire \\
\hline 4 & $30^{*}$ & -34 & Wire & Wire & Wire & Wire & Wire & Wire \\
\hline 5 & $150 \S$ & -48 & Wire & Wire & Wire & Wire & Wire & Wire \\
\hline 6 & $\mathrm{n} / \mathrm{i}$ & $\mathrm{n} / \mathrm{i}$ & Wire & Wire & Wire & Wire & Wire & Wire \\
\hline 7 & Distal & Distal & Distal & Distal & Wire & Wire & Wire & Wire \\
\hline 8 & $\mathrm{n} / \mathrm{i}$ & -10 & $10 \S$ & $5 \S$ & $35 \S$ & $-24 \S$ & Wire & Wire \\
\hline 9 & $\mathrm{n} / \mathrm{i}$ & -19 & $\mathrm{n} / \mathrm{i}$ & $\mathrm{n} / \mathrm{i}$ & Wire & Wire & Wire & Wire \\
\hline 10 & $20^{*}$ & -17 & Wire & Wire & Wire & Wire & Wire & Wire \\
\hline 11 & $127 \S$ & -45 & $70 \S$ & $-44 \S$ & $36^{*}$ & $-45^{*}$ & Wire & Wire \\
\hline 12 & $60 \S$ & -35 & $59^{*}$ & -35 & $29^{*}$ & $-31^{*}$ & Wire & Wire \\
\hline 13 & $\mathrm{n} / \mathrm{i}$ & $\mathrm{n} / \mathrm{i}$ & $94 \S$ & $-36 \S$ & Wire & Wire & Wire & Wire \\
\hline 14 & $58 \S$ & -31 & $125 \S$ & $-39 \S$ & $110 \S$ & $-43 \S$ & $22^{*}$ & $-21^{*}$ \\
\hline 15 & $\mathrm{n} / \mathrm{i}$ & $\mathrm{n} / \mathrm{i}$ & $50 \S$ & $-35 \S$ & Wire & Wire & Wire & Wire \\
\hline 16 & $270 \S$ & -43 & $44^{*}$ & -30 & Distal & Distal & Distal & Distal \\
\hline 17 & $29^{*}$ & -30 & $143 \S$ & $-36 \S$ & $19^{*}$ & -16 & Wire & Wire \\
\hline 18 & $77 \S$ & -31 & $63^{*}$ & -33 & $24^{*}$ & -24 & Wire & Wire \\
\hline MEAN & 74 & -38 & 69 & -32 & 53 & -35 & 19 & -21 \\
\hline STD & 75 & 10 & 41 & 14 & 40 & 15 & 4 & 1 \\
\hline
\end{tabular}

*sustained PVI, §̧nonsustained PVI, n/i—no PV isolation at first CB application, distal—no tracing due to distal SC position, wire—insufficient mechanical guidance of $\mathrm{SC}$ requiring change to regular guide wire. 
TABLE 3

Primary Endpoint: Feasibility of PVI with Spiral Catheter

\begin{tabular}{lcccc}
\hline Patient (\#) & LSPV & LIPV & RSPV & RIPV \\
\hline 1 & SC & Wire & SC & SC \\
2 & SC & SC & SC & Wire \\
3 & SC & Wire & Wire & Wire \\
4 & SC & Wire & Wire & Wire \\
5 & SC & Wire & Wire & Wire \\
6 & Wire & Wire & Wire & Wire \\
7 & SC & SC & Wire & Wire \\
8 & SC & SC & Wire & Wire \\
9 & SC & Wire & Wire & Wire \\
10 & SC & Wire & Wire & Wire \\
11 & SC & SC & SC & Wire \\
12 & SC & SC & SC & Wire \\
13 & SC & SC & Wire & Wire \\
14 & SC & SC & SC & SC \\
15 & SC & SC & Wire & Wire \\
16 & SC & SC & SC & SC \\
17 & SC & SC & SC & Wire \\
18 & SC & SC & SC & Wire \\
Total PVI with & $17^{*}(94 \%)$ & $11^{*}(61 \%)$ & $8^{*}(44 \%)$ & $3^{*}(17 \%)$ \\
SC (n; $\%)$ & & & & \\
Total PVI with & $1(6 \%)$ & $7(39 \%)$ & $10(66 \%)$ & $15(83 \%)$ \\
wire (n; $\%)$ & & & & \\
Total PVI (n; $\%)$ & $18(100 \%)$ & $18(100 \%)$ & $18(100 \%)$ & $18(100 \%)$ \\
\hline
\end{tabular}

${ }^{*} P<0.001$; non parametric Friedmann test. PVI $=$ pulmonary vein isolation; $\mathrm{SC}=$ spiral catheter; $\mathrm{LSPV}=$ left superior pulmonary vein; LIPV $=$ left inferior pulmonary vein; RSPV $=$ right superior pulmonary vein; RIPV = right inferior pulmonary vein.

\section{Discussion}

PVI is the cornerstone for catheter ablation of $\mathrm{AF}^{14}$ Cryothermal energy delivery via a balloon catheter represents a novel ablation strategy with the potential to achieve acute PVI with a single application. ${ }^{7-10}$ Using a solid-tip cryocatheter, real-time registration of local electrograms during the freezing period is complicated by ice ball formationinduced artefacts, prohibiting proper signal evaluation. ${ }^{15}$ The same limitation of nonavailability of real-time registration of LA-PV conduction applies for cryoballoon ablation and may have accounted for prolonged procedure times. So far, LA$\mathrm{PV}$ conduction properties during the cryoballoon freeze have remained completely unknown. Therefore, more information regarding real-time LA-PV conduction during cryoballoon ablation is clearly warranted. So far, cryoballoon PVI failure has been documented only after having accomplished fulltime application. If LA-PV conduction visualization reveals long time to PVI, this may indicate incomplete lesions that may be associated with increased PV conduction recovery. A tool that combines recording and guide wire features may contribute to safely assess the endpoint of acute PVI without repeatedly exchanging Lasso and cryoballoon catheter through 1 single large transseptal LA sheath. ${ }^{9}$

\section{Spiral Catheter as a Guide Wire for Cryoballoon Pulmonary Vein Isolation}

The primary endpoint of successful PVI using the cryoballoon in conjunction with the SC was achieved in 39 of 72 (54\%) PVs. Our initial experience demonstrates feasibility of the concept of real-time PV conduction registration during a
TABLE 4

Secondary Endpoint: Time to PV Conduction Block

\begin{tabular}{|c|c|c|c|c|}
\hline \multirow[b]{2}{*}{ \# PV } & \multicolumn{2}{|c|}{ Sustained PV Isolation } & \multicolumn{2}{|c|}{ Nonsustained PV Isolation } \\
\hline & $\begin{array}{l}\text { Time to PV } \\
\text { Block (s) }\end{array}$ & $\begin{array}{c}\text { Temperature } \\
\left({ }^{\circ} \mathbf{C}\right)\end{array}$ & $\begin{array}{l}\text { Time to PV } \\
\text { Block (s) }\end{array}$ & $\begin{array}{c}\text { Temperature } \\
\left({ }^{\circ} \mathbf{C}\right)\end{array}$ \\
\hline 1 & 20 & -54 & 150 & -48 \\
\hline 2 & 29 & -40 & 127 & -45 \\
\hline 3 & 18 & -43 & 60 & -35 \\
\hline 4 & 30 & -34 & 58 & -31 \\
\hline 5 & 20 & -17 & 50 & -35 \\
\hline 6 & 33 & -40 & 10 & 5 \\
\hline 7 & 95 & -54 & 70 & -44 \\
\hline 8 & 11 & -14 & 143 & -36 \\
\hline 9 & 36 & -45 & 94 & -36 \\
\hline 10 & 29 & -31 & 125 & -39 \\
\hline 11 & 22 & -21 & 35 & -24 \\
\hline 12 & 16 & -20 & 110 & -43 \\
\hline 13 & 44 & -30 & 270 & -43 \\
\hline 14 & 29 & -30 & 77 & -31 \\
\hline 15 & 59 & -35 & - & - \\
\hline 16 & 63 & -33 & - & - \\
\hline 17 & 19 & -16 & - & - \\
\hline 18 & 24 & -24 & - & - \\
\hline $\begin{array}{l}\text { MEAN } \pm \\
\text { STD }\end{array}$ & $33^{*} \pm 21$ & $-32 \pm 12(\mathrm{~ns})$ & $99^{*} \pm 65$ & $-35 \pm 13(\mathrm{~ns})$ \\
\hline
\end{tabular}

${ }^{*} P=0.002 . \mathrm{ns}-$ not significant, t-test. $\mathrm{PV}=$ pulmonary vein.

cryoballoon freeze. Whereas, LSPV isolation was relatively easy to accomplish utilizing the combination of cryoballoon and SC (17 of 18 PVs, 94\%) (Figs. 2A and 3A), isolation of the remaining PVs proved to be significantly more challenging (Table 3). As previously shown, ${ }^{16}$ the LSPV is the only PV that enters the LA from a left anterior direction and is therefore in direct alignment with the balloon/sheath system after transseptal LA puncture leading to a stable cryoballoon position at the LSPV ostium without distortion of the soft SC tip (Fig. 2A). This is in agreement with our observation, showing the significantly highest success rate for SC guided PVI at the LPSV (Table 3). In contrast, due to LA geometry all remaining PVs are not in alignment with the transseptal sheath after accessing the $\mathrm{LA}^{16}$ leading to increased rates of SC PVI failure or switching to a conventional stiff guide wire (Table 3, Fig. 1). Positioning the cryoballoon at the RSPV requires a clockwise rotation and firm forward pushing forces of the balloon/sheath system, distorting the soft distal part of the SC, resulting in nonstable balloon positions. Recently described cryoballoon ablation techniques ${ }^{9}$ for PVs that are not in alignment rely on a stiff guide wire. Our data demonstrated that the current soft SC cannot be used for all PVs instead of a stiff guide wire. Nonavailability of strong mechanical guide wire properties is the major limitation of the current SC version (Fig. 2B). Notably, this first generation SC with a very soft, nontraumatic distal tip was originally designed for the HIFU balloon and not for the cryoballoon. The HIFU balloon system does not rely on direct balloon/LA-PV tissue contact, ${ }^{12}$ hence strong mechanical guide wire support is not a prerequisite. In contrast, direct cryoballoon/LA-PV tissue contact is essential for cryoballoon lesion formation. ${ }^{7-10}$ The current SC provides less mechanical support than the conventional guide wire but causing no procedural complications. Future modification, for example, reinforcement of the distal catheter shaft may overcome this limitation, further 

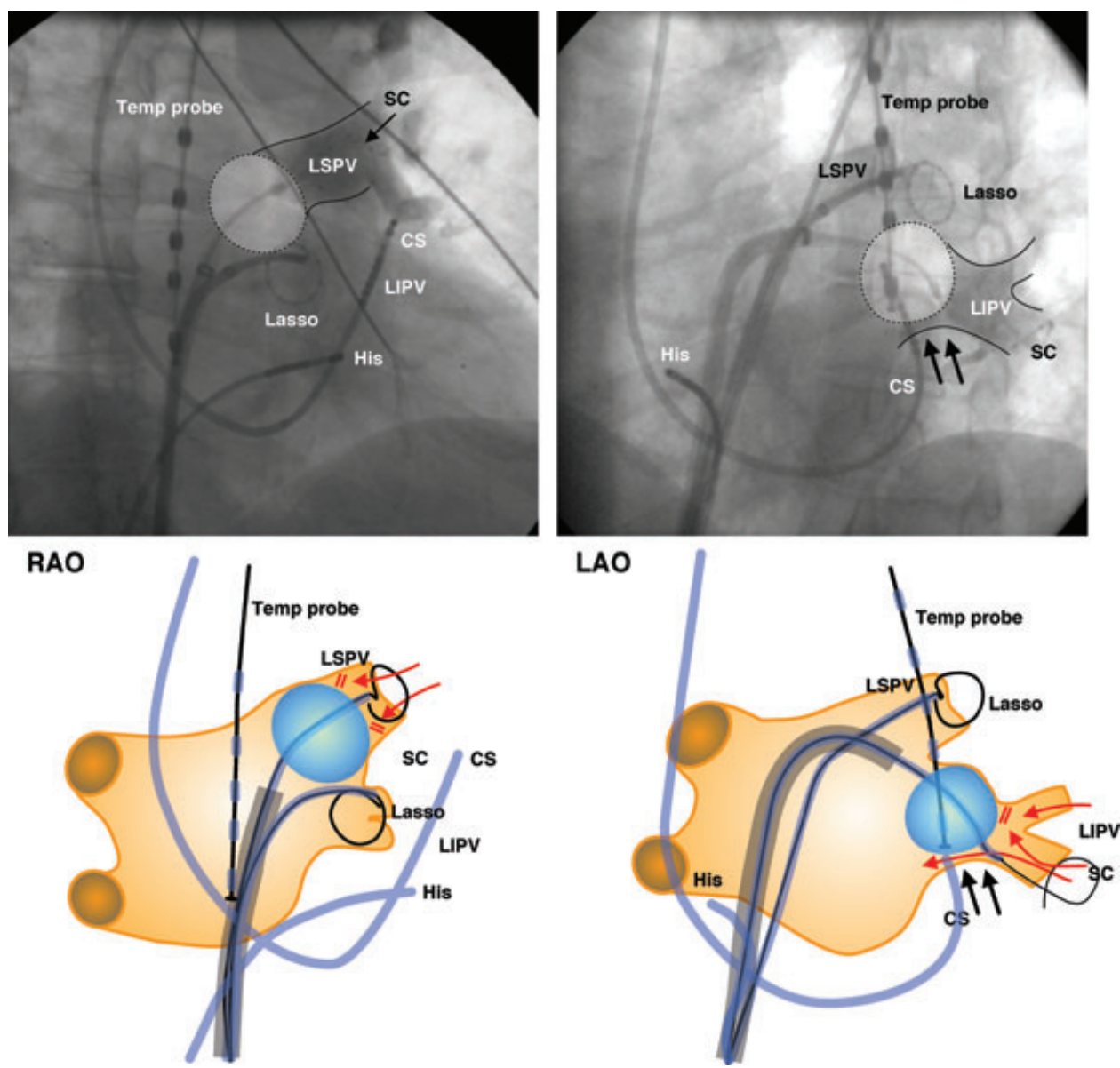

Figure 2. A: (Right panel) Before starting the freeze, complete PV occlusion of the LSPV as seen on this angiogram (RAO) utilizing the novel soft SC for cryoballoon guiding and real-time LA-PV conduction recording. No distortion of the SC in the LSPV because of direct alignment with the transseptal LA access. The corresponding cartoon (below) illustrates the fluoroscopic image. B: (Left panel) Before starting the freeze, incomplete PV occlusion of the LIPV (inferior gap, arrows) as seen on this angiogram (LAO) utilizing the soft SC for cryoballoon guiding and real-time LA-PV conduction recording. Distortion of the soft distal SC placed distally inside the LIPV because of no alignment with the transseptal LA access. The corresponding cartoon (below) illustrates the fluoroscopic image. $S C=$ spiral catheter; $C S=$ coronary sinus; His $=$ His bundle area; $L S P V=$ left superior pulmonary vein; LIPV $=$ left inferior pulmonary vein; Temp probe $=$ temperature probe in esophageus; $R A O=$ right anterior oblique; $L A O=$ left anterior oblique.

simplifying cryoballoon ablation, eventually allowing for a single transseptal puncture procedure.

\section{PV Conduction Block Visualized by the Spiral Catheter}

For the first time, assessment of real-time PV conduction during a cryoballoon freeze and precise determination of the moment of PV conduction block was possible using the novel SC enhancing the knowledge of cryoballoon lesion formation.

Real-time PV potential assessment was not possible in a subset of PVs because of switching to the conventional guide wire in PVs requiring more mechanical backup for a stable balloon position. Furthermore, real-time PV potential recording could not always be obtained due to SC positions distal to the level of PV muscular sleeves because: (1) the cryoballoon is mounted on a nose of 10$\mathrm{mm}$ length not allowing for proximal PV recording sites and (2) improving mechanical support required a distal SC position.

Cryoballoon temperatures do not directly reflect lesion size and did not differ at the moment of PVI in our se- ries. For focal cryocatheters it has been established that time to cryocatheter rewarming rather than absolute minimal electrode temperature is a better predictor for lesion size. ${ }^{17}$

Interestingly, our data demonstrated that rapid PV conduction block was associated with sustained PVI, whereas a prolonged time interval to block was associated with nonsustained PVI and PV reconnection during the waiting period. The phenomenon that time to conduction block predicted sustained conduction block has previously been observed for RFC energy application during accessory pathway ablation. ${ }^{18}$ In addition, real-time visualization of PV potential changes during the freezing period may result in online identification of residual conduction gaps allowing for readjustment of the balloon position during energy delivery as described empirically for the inferior PVs (pull down technique). ${ }^{9}$ Whether immediate successful PVI indicates improved chronic cryothermal energy lesions is debatable and will need to be determined in future studies. Furthermore, according to our data failure to achieve PVI during the first 99 seconds of cryoballoon energy delivery may alert the operator to reposition the cryoballoon rather than continuing a 


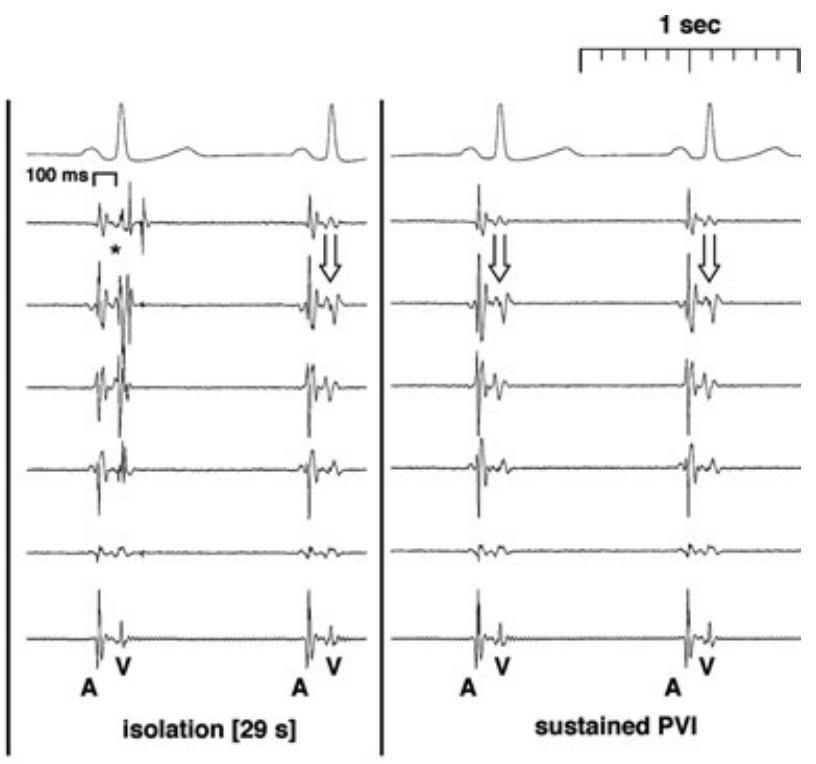

B
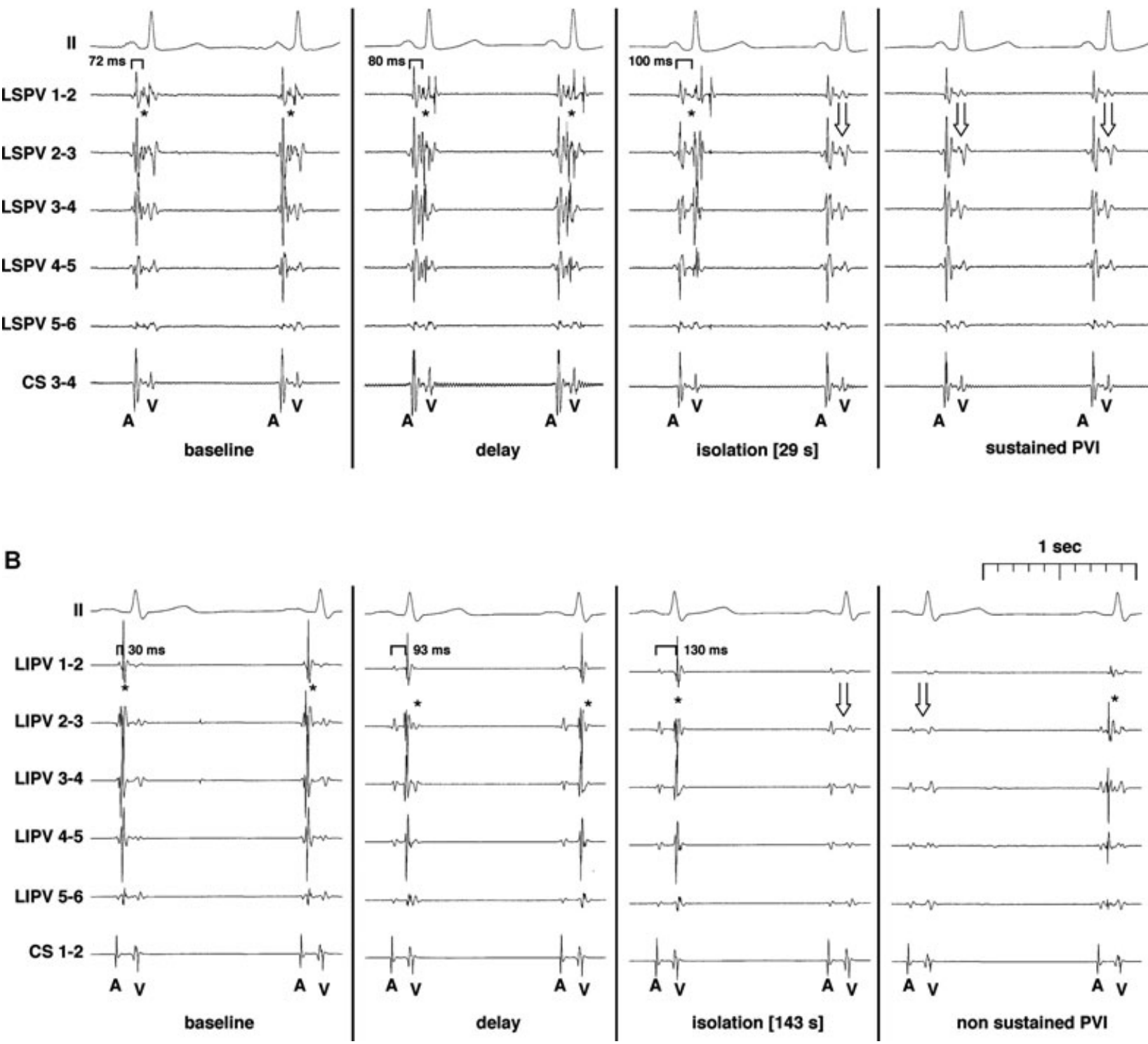

Figure 3. A: Corresponding PV signal recordings $\left(^{*}\right)$ obtained from the SC (poles 1-6) in the LSPV at baseline conditions (baseline). Separation of atrial farfield and $P V$ spike $\left(^{*}\right)$ during crythothermal energy application (delay) and successful $P V$ spike elimination (arrow) (isolation), which sustained isolated (arrow). B: Corresponding PV signal recordings (*) obtained from the SC (poles 1-6) in the LIPV at baseline conditions. Separation of atrial farfield and $P V$ spike (*) during the freezing period (delay) and successful PV spike elimination after 143 seconds (arrow) (isolation) and conduction recovery during the thawing phase (nonsustained $P V I *)$. A = atrium; $V=$ ventricle.

potentially unsuccessful ablation attempt. Accordingly, energy application to noncritical regions as well as procedure times may be reduced by early termination of unsuccessful cryoballoon applications.

\section{Limitations}

This study enrolled a small number of patients; consequently, a limited number of PVs and only acute procedural data could be systematically analyzed. Our observation that a short time to PVI predicts sustained PVI needs validation in larger future studies. The small number of PVs and patients analyzed precludes the conclusion that a short time to $\mathrm{PV}$ conduction block correlates with a decrease in $\mathrm{AF}$ recurrence.

\section{Conclusion}

Visualization of real-time PV conduction during cryoballoon freezing is safe, feasible, and allows accurate assessment of PVI onset in a subset of PVs. Time to PV conduction block predicts sustained PVI. However, modification of the current $\mathrm{SC} /$ cryoballoon design is a prerequisite in order to further simplify cryoballoon PVI.

\section{References}

1. Fuster V, Ryden LE, Cannom DS, Crijns HJ, Curtis AB, Ellenbogen KA, Halperin JL, Le Heuzey JY, Kay GN, Lowe JE, Olsson SB, Prystowsky EN, Tamargo JL, Wann S, Smith SC Jr, Jacobs AK, Adams $\mathrm{CD}$, Anderson JL, Antman EM, Halperin JL, Hunt SA, Nishimura R, Ornato JP, Page RL, Riegel B, Priori SG, Blanc JJ, Budaj A, Camm 
AJ, Dean V, Deckers JW, Despres C, Dickstein K, Lekakis J, McGregor K, Metra M, Morais J, Osterspey A, Tamargo JL, Zamorano JL, ACC/AHA/ESC 2006: Guidelines for the Management of Patients with Atrial Fibrillation: A report of the American College of Cardiology/American Heart Association Task Force on Practice Guidelines and the European Society of Cardiology Committee for Practice Guidelines (Writing Committee to Revise the 2001 Guidelines for the Management of Patients with Atrial Fibrillation): Developed in collaboration with the European Heart Rhythm Association and the Heart Rhythm Society. Circulation 2006; 114:e257-e354.

2. Ouyang F, Bansch D, Ernst S, Schaumann A, Hachiya H, Chen M, Chun J, Falk P, Khanedani A, Antz M, Kuck KH: Complete isolation of left atrium surrounding the pulmonary veins: New insights from the double-Lasso technique in paroxysmal atrial fibrillation. Circulation 2004;110:2090-2096.

3. Robbins IM, Colvin EV, Doyle TP, Kemp WE, Loyd JE, McMahon WS, Kay GN: Pulmonary vein stenosis after catheter ablation of atrial fibrillation. Circulation 1998;98:1769-1775.

4. Mesas CE, Pappone C, Lang CC, Gugliotta F, Tomita T, Vicedomini G, Sala S, Paglino G, Gulletta S, Ferro A, Santinelli V: Left atrial tachycardia after circumferential pulmonary vein ablation for atrial fibrillation: Electroanatomic characterization and treatment. J Am Coll Cardiol 2004;44:1071-1079.

5. Cappato R, Calkins H, Chen SA, Davies W, Iesaka Y, Kalman J, Kim YH, Klein G, Packer D, Skanes A: Worldwide survey on the methods, efficacy, and safety of catheter ablation for human atrial fibrillation. Circulation 2005;111(9):1100-1105.

6. Van Belle Y, Janse P, Rivero-Ayerza MJ, Thornton AS, Jessurun ER, Theuns D, Jordaens L: Pulmonary vein isolation using an occluding cryoballoon for circumferential ablation: Feasibility, complications, and short-term outcome. Eur Heart J 2007;28(18):2231-2237.

7. Klein G, Oswald H, Gardiwal A, Lusebrink U, Lissel C, Yu H, Drexler $\mathrm{H}$ : Efficacy of pulmonary vein isolation by cryoballoon ablation in patients with paroxysmal atrial fibrillation. Heart Rhythm 2008;5:802806.

8. Chun KR, Schmidt B, Metzner A, Tilz R, Zerm T, Koster I, Furnkranz A, Koektuerk B, Konstantinidou M, Antz M, Ouyang F, Kuck KH: The 'single big cryoballoon' technique for acute pulmonary vein isolation in patients with paroxysmal atrial fibrillation: A prospective observational single centre study. Eur Heart J 2009;30:699-709.

9. Neumann T, Vogt J, Schumacher B, Dorszewski A, Kuniss M, Neuser
H, Kurzidim K, Berkowitsch A, Koller M, Heintze J, Scholz U, Wetzel U, Schneider MA, Horstkotte D, Hamm CW, Pitschner HF: Circumferential pulmonary vein isolation with the cryoballoon technique results from a prospective 3-center study. J Am Coll Cardiol 2008;52:273-278.

10. Chun KJ, Ouyang F, Schmidt B, Kuck KH: Focal atrial tachycardia originating from the right atrial appendage: First successful cryoballoon isolation. J Cardiovasc Electrophysiol 2009;20:338-341.

11. Schmidt B, Antz M, Ernst S, Ouyang F, Falk P, Chun JK, Kuck KH: Pulmonary vein isolation by high-intensity focused ultrasound: First-inman study with a steerable balloon catheter. Heart Rhythm 2007;4:575584 .

12. Marom EM, Herndon JE, Kim YH, McAdams HP: Variations in pulmonary venous drainage to the left atrium: Implications for radiofrequency ablation. Radiology 2004;230:824-829.

13. Calkins H, Brugada J, Packer DL, Cappato R, Chen SA, Crijns HJ, Damiano RJ Jr, Davies DW, Haines DE, Haissaguerre M, Iesaka Y, Jackman W, Jais P, Kottkamp H, Kuck KH, Lindsay BD, Marchlinski FE, McCarthy PM, Mont JL, Morady F, Nademanee K, Natale A, Pappone C, Prystowsky E, Raviele A, Ruskin JN, Shemin RJ: HRS/EHRA/ECAS expert Consensus Statement on catheter and surgical ablation of atrial fibrillation: Recommendations for personnel, policy, procedures and follow-up. A report of the Heart Rhythm Society (HRS) Task Force on catheter and surgical ablation of atrial fibrillation. Heart Rhythm 2007;4:816-861.

14. Skanes AC, Dubuc M, Klein GJ, Thibault B, Krahn AD, Yee R, Roy D, Guerra P, Talajic M: Cryothermal ablation of the slow pathway for the elimination of atrioventricular nodal reentrant tachycardia. Circulation 2000;102:2856-2860.

15. Schmidt B, Ernst S, Ouyang F, Chun KR, Broemel T, Bansch D, Kuck $\mathrm{KH}$, Antz M: External and endoluminal analysis of left atrial anatomy and the pulmonary veins in three-dimensional reconstructions of magnetic resonance angiography: The full insight from inside. J Cardiovasc Electrophysiol 2006;17:957-964.

16. Parvez B, Goldberg SM, Pathak V, Schubert CM, Wood MA: Time to electrode rewarming after cryoablation predicts lesion size. J Cardiovasc Electrophysiol 2007;18:845-848.

17. Twidale N, Wang XZ, Beckman KJ, McClelland JH, Moulton KP, Prior MI, Hazlitt HA, Lazzara R, Jackman WM: Factors associated with recurrence of accessory pathway conduction after radiofrequency catheter ablation. Pacing Clin Electrophysiol 1991;14(11 Pt 2):20422048. 